PROCEEDINGS OF THE

AMERICAN MATHEMATICAL SOCIETY

Volume 138, Number 6, June 2010, Pages 2065-2070

S 0002-9939(10)10219-6

Article electronically published on January 7, 2010

\title{
ON MAXIMAL INJECTIVE SUBALGEBRAS
}

\author{
MINGCHU GAO
}

(Communicated by Marius Junge)

\begin{abstract}
Let $\mathcal{A}_{i}$ be a type $I$ von Neumann subalgebra in a type $I I_{1}$ factor $\mathcal{M}_{i}$ with the faithful trace $\tau_{i}$ such that $\mathcal{A}_{i}^{\prime} \cap \mathcal{M}_{i} \subseteq \mathcal{A}_{i}$, for $i=1,2, \cdots$. Moreover, suppose $\mathcal{A}_{i}$ has the asymptotically orthogonal property in $\mathcal{M}_{i}$ after tensoring the finite von Neumann algebra $\otimes_{j \neq i} \mathcal{M}_{j}$, for all $i=1,2, \cdots$. Then we show that $\otimes_{i=1}^{\infty} \mathcal{A}_{i}$ is maximal injective in the infinite tensor product von Neumann algebra $\otimes_{i=1}^{\infty} \mathcal{M}_{i}$. As a consequence, we get the following result. Let $\left\{\mathbb{F}_{n_{i}} ; i=1,2, \cdots\right\}$ be a sequence of free groups with $n_{i}(>1)$ generators. Let $\mathcal{A}_{i}$ be the masa of group von Neumann algebra $\mathcal{L}_{\mathbb{F}_{n_{i}}}$ generated by a generator of $\mathbb{F}_{n_{i}}$ or by the sum of all generators and their inverses of the group. Then $\otimes_{i=1}^{\infty} \mathcal{A}_{i}$ is maximal injective in the infinite tensor product von Neumann algebra $\otimes_{i=1}^{\infty} \mathcal{L}_{\mathbb{F}_{n_{i}}}$.
\end{abstract}

\section{INTRODUCTION}

A von Neumann subalgebra $\mathcal{B}$ of a von Neumann algebra $\mathcal{M}$ is a maximal injective subalgebra of $\mathcal{N}$ if $\mathcal{B}$ is injective and cannot be included properly in an injective von Neumann subalgebra $\mathcal{L}$ of $\mathcal{M}$. The injective von Neumann subalgebras of a type $I I_{1}$ factor are closed under weak limits of unions of ascending chains so maximal injective von Neumann subalgebras exist in all type $I I_{1}$ factors $([\mathrm{Co}]$ ). But it is quite difficult to find explicit examples of maximal injective subalgebras in type $I I_{1}$ factors. Popa $[\mathrm{Po}$ found firstly explicit examples of maximal injective von Neumann algebras in type $I I_{1}$ factors by showing that the masa (i.e., maximal abelian self-adjoint subalgebra) of $\mathcal{L}_{\mathbb{F}_{n}}$ generated a generator of the free group $\mathbb{F}_{n}$ of $n$ generators, which is called a generator masa, is maximal injective in the free group factor $\mathcal{L}_{\mathbb{F}_{n}}$. Popa's work $[\mathrm{Po}$ also answered the following Kadision's problem negatively.

Kadison's problem ( $\mathrm{Ka}$ and $\mathrm{Ge})$. Does each self-adjoint operator in a $I I_{1}$ factor lie in some hyperfinite subfactor?

Ge Ge1 provided plenty of examples of maximal injective subalgebras in type $I I_{1}$ factors by showing that every non-atomic injective finite von Neumann algebra can be embedded as a maximal injective subalgebra in the free product von Neumann algebra of the algebra and the group von Neumann algebra of a countable

Received by the editors March 16, 2009, and, in revised form, September 20, 2009.

2010 Mathematics Subject Classification. Primary 46L10.

Key words and phrases. Finite von Neumann algebras, maximal injective subalgebras, tensor products, free group von Neumann algebras.

(C)2010 American Mathematical Society Reverts to public domain 28 years from publication 
discrete group. Shen Sh found a McDuff factor with a masa as its maximal injective subalgebra. Hou [Hou found a non-McDuff type $I I_{1}$ factor of property $\Gamma$, where a masa is a maximal injective subalgebra.

In the paper $[\mathrm{Po}]$, Popa asked the following question.

Popa's problem. Suppose $\mathcal{B}_{i} \subset \mathcal{M}_{i}(i=1,2)$ are two inclusions of von Neumann algebras with $\mathcal{B}_{i}$ a maximal injective von Neumann subalgebra of $\mathcal{M}_{i}$. Must $\mathcal{B}_{1} \otimes \mathcal{B}_{2}$ be maximal injective in $\mathcal{M}_{1} \otimes \mathcal{M}_{2}$ ?

The first progress on this problem was made by Ge and Kadison, who gave a positive answer in GK when $\mathcal{B}_{1}=\mathcal{M}_{1}$ is an injective factor. The result was subsequently improved by Stratila and Zsido in [SZ, who showed that if $\mathcal{B}_{1}=\mathcal{M}_{1}$ is an injective finite von Neumann algebra and $\mathcal{M}_{2}$ has a separable predual, then $\mathcal{M}_{1} \otimes \mathcal{B}_{2}$ is maximal injective in $\mathcal{M}_{1} \otimes \mathcal{M}_{2}$. Shen $[\mathrm{Sh}$ investigated the problem for generator masas in free group factors and showed that the (infinite or finite) tensor product of generator masas of free group von Neumann algebras is maximal injective in the tensor product of the group von Neumann algebras (Theorem 4.1 in $[\mathrm{Sh}])$. Fang [ $\mathrm{Fa}$ abtained a positive answer to Popa's question when the center of $\mathcal{B}_{1}$ is atomic and $\mathcal{M}_{2}$ has a separable predual. Very recently, Cameron, Fang, Ravichandran, and White [CFRW] showed that the radical masa (i.e., the masa generated by the sum of all generators and their inverses of the group) in the free group von Neumann algebra $\mathcal{L}_{F_{n}}$ is maximal injective (Theorems 2.8 and 6.2 in CFRW]). They also proved that the finite tensor product of generator and radical masas is maximal injective in the finite tensor product of the group von Neumann algebras (Corollary 6.5 in [CFRW]).

Let $\mathcal{A}_{i}$ be a type $I$ von Neumann subalgebra in a type $I I_{1}$ factor $\mathcal{M}_{i}$ with the faithful trace $\tau_{i}$ such that $\mathcal{A}_{i}^{\prime} \cap \mathcal{M}_{i} \subseteq \mathcal{A}_{i}$, for $i=1,2, \cdots$. Moreover, suppose $\mathcal{A}_{i}$ has the asymptotically orthogonal property in $\mathcal{M}_{i}$ after tensoring the finite von Neumann algebra $\otimes_{j \neq i} \mathcal{M}_{j}$, for all $i=1,2, \cdots$ (see Definition 2.3). Then we show in this paper that $\otimes_{i=1}^{\infty} \mathcal{A}_{i}$ is maximal injective in the infinite tensor product von Neumann algebra $\otimes_{i=1}^{\infty} \mathcal{M}_{i}$. As a consequence, we get the following result: The infinite tensor product of generator and radical masas is maximal injective in the infinite tensor product of the free group von Neumann algebras. This result generalizes the results in Sh and CFRW].

In this paper, we use $\mathcal{M} \otimes \mathcal{N}$ to denote the von Neumann algebra tensor product of two von Neumann algebras $\mathcal{M}$ and $\mathcal{N}$. We assume that all von Neumann algebras (except ultra-power von Neumann algebras) have separable preduals.

\section{The MAIN RESUlts}

Lemma 2.1. Let $\mathcal{A}_{i}$ be a von Neumann subalgebra in a finite von Neumann algebra $\mathcal{M}$ with a faithful normal tracial state $\tau_{i}$, for $1=1,2, \cdots$. Then

$$
\left(\otimes_{i=1}^{\infty} A_{i}\right)^{\prime} \cap \otimes_{i=1}^{\infty} \mathcal{M}_{i}=\otimes_{i=1}^{\infty}\left(\mathcal{A}_{i}^{\prime} \cap \mathcal{M}_{i}\right) .
$$

Proof. Let us denote $\otimes_{i=1}^{\infty} \mathcal{M}_{i}$ by $\mathcal{M}$. By Exercise 12.4.37 in KR (see also Lemma 1.5 in $[\mathrm{Fa}]$ ) and induction,

$$
\left(\otimes_{1 \leq i \leq n} \mathcal{A}_{i}\right)^{\prime} \cap\left(\otimes_{1 \leq i \leq n} \mathcal{M}_{i}\right)=\otimes_{1 \leq i \leq n} \mathcal{A}_{i}^{\prime} \cap \mathcal{M}_{i}
$$

for all $n$. By the construction of $\mathcal{M}=\otimes_{i=1}^{\infty} \mathcal{M}_{i}$, we may identify $\mathcal{M}_{i}$ with its image in $\otimes_{i=1}^{\infty} \mathcal{M}_{i}$ as an algebra and its image in the acting Hilbert space $\otimes_{n=1}^{\infty} L^{2}\left(\mathcal{M}_{n}, \tau_{n}\right)$ as an inner product space. Hence, we may regard $\mathcal{M}_{1}, \mathcal{M}_{2}, \cdots$ as commutative 
von Neumann subalgebras of $\mathcal{M}$ and inner-product subspaces of Hilbert space $L^{2}(M, \tau)=\otimes_{i=1}^{\infty} L^{2}\left(\mathcal{M}_{i}, \tau_{i}\right)$. Then $\bigcup_{n>1}\left(\otimes_{i \leq n} \mathcal{M}_{i}\right)$ is a strong operator dense ${ }^{*}$-subalgebra of $\otimes_{i=1}^{\infty} \mathcal{M}_{i}$. Let $E_{n}: \mathcal{M} \rightarrow \otimes_{i \leq n} \mathcal{M}_{i}$ be the trace-preserving conditional expectation onto $\otimes_{i \leq n} \mathcal{M}_{i}$. For $x \in \otimes_{i=1}^{\infty}\left(\mathcal{A}_{i}^{\prime} \cap \mathcal{M}_{i}\right) \subseteq \mathcal{M}$, by the Kaplansky density theorem, there is a sequence $\left(x_{n}\right)_{n}$ of elements in

$$
\bigcup_{n \geq 1}\left(\left(\otimes_{i \leq n} \mathcal{A}_{i}\right)^{\prime} \cap \otimes_{i \leq n} \mathcal{M}_{i}\right)=\bigcup_{n \geq 1}\left(\otimes_{1 \leq i \leq n} \mathcal{A}_{i}^{\prime} \cap \mathcal{M}_{i}\right)
$$

such that $\lim _{n \rightarrow \infty}\left\|x-x_{n}\right\|_{2}=0$. Obviously, we may choose $x_{n} \in\left(\otimes_{i \leq n} \mathcal{A}_{i}\right)^{\prime} \cap$ $\left(\otimes_{i \leq n} \mathcal{M}_{i}\right)$. Thus, for every $i$ and $y \in \mathcal{A}_{i}$, we have $x_{n} y=y x_{n}$, when $n>i$. It follows that

$$
\|x y-y x\|_{2}=\lim _{n \rightarrow \infty}\left\|x_{n} y-y x_{n}\right\|_{2}=0 .
$$

Hence, $x \in\left(\otimes_{i=1}^{\infty} \mathcal{A}_{i}\right)^{\prime} \cap \mathcal{M}$. Conversely, for $x \in\left(\otimes_{i=1}^{\infty} \mathcal{A}_{i}\right)^{\prime} \cap \mathcal{M}$ and $y \in \otimes_{i \leq n} \mathcal{A}_{i}$, $n=1,2, \cdots$, we have

$$
E_{n}(x y)=E_{n}(x) y=E_{n}(y x)=y E_{n}(x)
$$

thus,

$$
E_{n}(x) \in\left(\otimes_{i \leq n} \mathcal{A}_{i}\right)^{\prime} \cap \otimes_{i \leq n} \mathcal{M}_{i}=\otimes_{i \leq n}\left(\mathcal{A}_{i}^{\prime} \cap \mathcal{M}_{i}\right) \subseteq \otimes_{i=1}^{\infty}\left(\mathcal{A}_{i}^{\prime} \cap \mathcal{M}_{i}\right) .
$$

Now we show that $E_{n}(x) \rightarrow x$ with respect to the strong operator topology, for $x \in \mathcal{M}$. This may be a well known result. We give a proof here for completeness. After identifying $\mathcal{M}_{1} \otimes \cdots \otimes \mathcal{M}_{n}$ with its image in $\mathcal{M}:=\otimes_{i=1}^{\infty} \mathcal{M}_{i}$, the space $\mathcal{M}_{0}:=\bigcup_{n \geq 1} \otimes_{1 \leq i \leq n} \mathcal{M}_{i}$ is a strong operator dense ${ }^{*}$-subalgebra of $\mathcal{M}$. By the Kaplansky density theorem, we can find a net $\left\{x_{\lambda}: \lambda \in \Lambda\right\}$ of elements in $\mathcal{M}_{0}$ such that $x_{\lambda} \rightarrow x$ in strong operator topology, and $\left\|x_{\lambda}\right\| \leq\|x\|$. Since $\mathcal{M}$ acts on $L^{2}(\mathcal{M}, \tau)$ standardly and $\mathcal{M}$ has a separable predual, we can find a sequence $\left\{x_{n}\right\}_{n}$ of elements in $\mathcal{M}_{0}$ such that $x_{n} \rightarrow x$ in strong operator topology (and in the Hilbert norm $\|\cdot\|_{2}$ ). For every $\epsilon>0$, we can find an $n$ such that $\left\|x-x_{n}\right\|_{2}<\epsilon / 2$. For $x_{n} \in \otimes_{i \leq l} \mathcal{M}_{i}$, we have $E_{k}\left(x_{n}\right)=x_{n}$, when $k>l$. Thus,

$$
\left\|x-E_{k}(x)\right\|_{2}=\left\|x-x_{n}-E_{k}\left(x-x_{n}\right)\right\|_{2}<\epsilon .
$$

Thus, $\lim _{k \rightarrow \infty}\left\|x-E_{k}(x)\right\|_{2}=0$. Hence, $E_{\otimes_{i \leq k} \mathcal{M}_{i}}(x) \rightarrow x$ in strong operator topology, since the convergence in strong operator topology is equivalent to that in $\|\cdot\|_{2}$ on norm bounded sets if $\mathcal{M}$ has a separating and generating element. Hence, we have $x \in \otimes_{i=1}^{\infty}\left(\mathcal{A}_{i}^{\prime} \cap \mathcal{M}_{i}\right)$, for all $x \in\left(\otimes_{i=1}^{\infty} \mathcal{A}_{i}\right)^{\prime} \cap \mathcal{M}$. The proof is complete.

As a consequence, we have the following result.

Corollary 2.2. Let $\mathcal{A}_{i} \subset \mathcal{M}_{i}$ be an inclusion of a masa in a finite von Neumann algebra $\mathcal{M}_{i}$ with a fixed faithful normal trace $\tau_{i}$, for $i=1,2, \ldots$ Then $\otimes_{i=1}^{\infty} \mathcal{A}_{i}$ is a masa in $\otimes_{i=1}^{\infty} \mathcal{M}_{i}$.

Cameron, Fang, Ravichandran, and White introduced the following concept in CFRW.

Definition 2.3 ([CFRW]). Let $\mathcal{A}$ be a type $I$ von Neumann subalgebra of a type $I I_{1}$ von Neumann algebra $\mathcal{M}$ with a fixed faithful normal trace $\tau_{\mathcal{M}}$. Let $\mathcal{N}$ be a finite von Neumann algebra with a fixed faithful trace $\tau_{\mathcal{N}}$. We say that $\mathcal{A}$ has the asymptotic orthogonality property after tensoring $\mathcal{N}$ if there is a non-principal ultrafilter $\omega \in \beta \mathbb{N}-\mathbb{N}$ such that $x_{1} y_{1} \perp y_{2} x_{2}$ whenever $x_{1}, x_{2} \in(\mathcal{A} \otimes \mathbb{C} 1)^{\prime} \cap(\mathcal{M} \bar{\otimes} \mathcal{N})^{\omega}$ with $E_{(\mathcal{A} \bar{\otimes} \mathcal{N}) \omega}\left(x_{i}\right)=0$ and $y_{i} \in \mathcal{M} \bar{\otimes} \mathcal{N}$ with $E_{\mathcal{A} \bar{\otimes} \mathcal{N}}\left(y_{i}\right)=0$, for $i=1$, 2. Especially, 
$\mathcal{A}$ has the asymptotic orthogonality property in $\mathcal{M}$ if it has the property in $\mathcal{M}$ after tensoring $\mathcal{N}=\mathbb{C} 1$. Here $E_{\mathcal{A}}: \mathcal{A} \rightarrow \mathcal{N}$ is the trace-preserving conditional expectation from a finite von Neumann algebra $\mathcal{M}$ with a fixed faithful normal trace onto its von Neumann subalgebra $\mathcal{A}$.

Remark 2.4. If a type $I$ von Neumann subalgebra $\mathcal{A}$ of a type $I I_{1}$ von Neumann algebra $\mathcal{M}$ has the asymptotic orthogonality property after tensoring a finite von Neumann algebra $\mathcal{N}$, and $\mathcal{N}_{1}$ and $\mathcal{N}_{2}$ are von Neumann subalgebras of $\mathcal{N}$ such that $\mathcal{N} \cong \mathcal{N}_{1} \otimes \mathcal{N}_{2}$, then $\mathcal{A}$ has the asymptotic orthogonality property after tensoring $\mathcal{N}_{1}$ or $\mathcal{N}_{2}$. Especially, $\mathcal{A}$ is asymptotic orthogonal in $\mathcal{M}$ (after tensoring $\mathbb{C} I$ ).

Proof. We prove the property for $\mathcal{N}_{1}$ only, since the same method works for $\mathcal{N}_{2}$. We may assume that $\mathcal{N}=\mathcal{N}_{1} \otimes \mathcal{N}_{2}$. The algebra $\left(\mathcal{M} \otimes \mathcal{N}_{1}\right)^{\omega}$ can be embedded into $(\mathcal{M} \otimes \mathcal{N})^{\omega}$ by the argument in $(2.3)$ of [Ge1]. Thus, we may consider $\left(\mathcal{M} \otimes \mathcal{N}_{1}\right)^{\omega} \subseteq$ $(\mathcal{M} \otimes \mathcal{N})^{\omega}$. Let

$$
x_{1}=\left(x_{1, n}\right)_{n}, x_{2}=\left(x_{2, n}\right)_{n} \in(\mathcal{A} \otimes \mathbb{C} I)^{\prime} \cap\left(\mathcal{M} \otimes \mathcal{N}_{1}\right)^{\omega}
$$

with $E_{\left(\mathcal{A} \otimes \mathcal{N}_{1}\right) \omega}\left(x_{i}\right)=0, i=1,2$. Let $y_{1}, y_{2} \in \mathcal{M} \otimes \mathcal{N}_{1}$ be such that $E_{\mathcal{A} \otimes \mathcal{N}_{1}}\left(y_{i}\right)=$ $0, i=1,2$. Let $\widetilde{x}_{i}=\left(x_{i, n} \otimes I\right)_{n} \in(\mathcal{A} \otimes \mathbb{C} I)^{\prime} \cap(\mathcal{M} \otimes \mathcal{N})^{\omega}$ and $\widetilde{y}_{i}=y_{i} \otimes I \in$ $\mathcal{M} \otimes \mathcal{N}, i=1,2$. Then we have

$$
\begin{aligned}
\tau_{\omega}\left(\left(E_{(\mathcal{A} \otimes \mathcal{N})^{\omega}}\left(\widetilde{x}_{i}\right)\right)^{*} E_{(\mathcal{A} \otimes \mathcal{N}) \omega}\left(\widetilde{x}_{i}\right)\right) & =\lim _{n \rightarrow \omega} \tau_{\mathcal{M} \otimes \mathcal{N}}\left(E_{\mathcal{A} \otimes \mathcal{N}}\left(x_{i, n} \otimes I\right)^{*} E_{\mathcal{A} \otimes \mathcal{N}}\left(x_{i, n} \otimes I\right)\right) \\
& =\lim _{n \rightarrow \omega} \tau_{\mathcal{M} \otimes \mathcal{N}_{1}}\left(\left(E_{\mathcal{A} \otimes \mathcal{N}_{1}}\left(x_{i, n}\right)\right)^{*}\left(E_{\mathcal{A} \otimes \mathcal{N}_{1}}\left(x_{i, n}\right)\right)\right) \\
& =\lim _{n \rightarrow \omega}\left\|E_{\mathcal{A} \otimes \mathcal{N}_{1}}\left(x_{i, n}\right)\right\|_{2}^{2} \\
& =\left\|\left(E_{\left(\mathcal{A} \otimes \mathcal{N}_{1}\right) \omega}\left(x_{i}\right)\right)\right\|_{2, \tau_{\omega}}^{2}=0
\end{aligned}
$$

where $\tau_{\mathcal{M} \otimes \mathcal{N}}=\tau_{\mathcal{M}} \otimes \tau_{\mathcal{N}}=\tau_{\mathcal{M}} \otimes \tau_{\mathcal{N}_{1}} \otimes \tau_{\mathcal{N}_{2}}$, and we used the fact that $E_{\mathcal{A} \otimes \mathcal{B}}=$ $E_{\mathcal{A}} \otimes E_{\mathcal{B}}$ for two inclusions $\mathcal{A} \subseteq \mathcal{M}$ and $\mathcal{B} \subseteq \mathcal{N}$ of finite von Neumann algebras (Remark 3.6.4 in [SS] $)$. Hence, $E_{(\mathcal{A} \otimes \mathcal{N})^{\omega}}\left(\widetilde{x}_{i}\right)=0, i=1$, 2. Similarly, $E_{\mathcal{A} \otimes \mathcal{N}}\left(\widetilde{y}_{i}\right)=0$. By the hypothesis,

$$
\begin{aligned}
0 & =\tau_{\omega}\left(\widetilde{x}_{2}^{*} \widetilde{y}_{2}^{*} \widetilde{x}_{1} \widetilde{y}_{1}\right) \\
& =\lim _{n \rightarrow \omega} \tau\left(\left(x_{2, n}^{*} \otimes I\right)\left(y_{2}^{*} \otimes I\right)\left(x_{1, n} \otimes I\right)\left(y_{1} \otimes I\right)\right) \\
& =\lim _{n \rightarrow \omega} \tau\left(x_{2, n}^{*} y_{2}^{*} x_{1, n}^{*} y_{1}\right) \\
& =\left\langle x_{1} y_{1}, y_{2} x_{2}\right\rangle_{\tau_{\omega}} .
\end{aligned}
$$

The proof is complete.

As a corollary (and a sort of generalization) of Theorem 2.8 in [CFRW], we have the following result. Let us first recall a concept from [SS and CFRW]. Let $\mathcal{B} \subset \mathcal{M}$ be an inclusion of von Neumann algebras with $\mathcal{B}^{\prime} \cap \mathcal{M} \subset \mathcal{B}$. A partial isometry $v \in \mathcal{M}$ is a groupoid normalizer of $\mathcal{B}$ if $v \mathcal{B} v^{*} \subset \mathcal{B}$ and $v^{*} \mathcal{B} v \subset \mathcal{B}$. We write $\mathcal{G N}_{\mathcal{M}}(\mathcal{B})$ for the collection of groupoid normalizers.

Lemma 2.5. Let $\mathcal{M}_{1}, \mathcal{M}_{2}, \cdots, \mathcal{M}_{n}$ be finite von Neumann algebras with a fixed faithful trace $\tau_{i}$ on $\mathcal{M}_{i}, i=1,2, \cdots, n$. Let $\mathcal{A}_{i}$ be a type I von Neumann subalgebra of $\mathcal{M}_{i}$ with $\mathcal{G N}_{\mathcal{M}_{i}}\left(\mathcal{A}_{i}\right) \subset \mathcal{A}_{i}$ and with the asymptotic orthogonality property after tensoring by $\otimes_{j \neq i} \mathcal{M}_{j}$, for $i=1,2, \cdots, n$. Then $\mathcal{A}_{i} \otimes \cdots \otimes \mathcal{A}_{n}$ is maximal injective in $\mathcal{M}=\mathcal{M}_{1} \otimes \cdots \otimes \mathcal{M}_{n}$. 
Proof. By the above remark, $\mathcal{A}_{i}$ is asymptotically orthogonal in $\mathcal{M}_{i}$, for all $i$. By Corollary 2.9 in [CFRW], $\mathcal{A}$ is maximal injective in $\mathcal{M}_{i}$, for all $i$. By Theorem 2.8 in [CFRW], $\mathcal{A}_{1} \otimes \mathcal{A}_{2}$ is maximal injective and of type $I$ in $\mathcal{M}_{1} \otimes \mathcal{M}_{2}$. By induction, $\mathcal{A}_{1} \otimes \cdots \otimes \mathcal{A}_{n}$ is maximal injective in $\mathcal{M}_{1} \otimes \cdots \otimes \mathcal{M}_{n}$.

We are in a position to give the main result of this paper. Some ideas in the proof are similar to those in [Sh].

Theorem 2.6. Let $\mathcal{A}_{i} \subseteq \mathcal{M}_{i}$ be an inclusion of a type I von Neumann subalgebra $\mathcal{A}_{i}$ in a type $I I_{1}$ factor $\mathcal{M}_{i}$ with the faithful normal trace $\tau_{i}, i=1,2, \cdots$. If $\mathcal{A}_{i}^{\prime} \cap$ $\mathcal{M}_{i} \subset \mathcal{A}_{i}$ and $\mathcal{A}_{i}$ has the asymptotic orthogonality property in $\mathcal{M}_{i}$ after tensoring the finite von Neumann algebra $\otimes_{j \neq i} \mathcal{M}_{j}$, for $i=1,2, \cdots$, then $\mathcal{A}=\otimes_{i=1}^{\infty} \mathcal{A}_{i}$ is a maximal injective subalgebra of von Neumann algebra $\mathcal{M}=\otimes_{i=1}^{\infty} \mathcal{M}_{i}$.

Proof. Let $\mathcal{B}_{n}=\left(\otimes_{i=1}^{n-1} \mathcal{M}_{i}\right) \otimes\left(\otimes_{i=n}^{\infty} \mathcal{A}_{i}\right)$. Then $\mathcal{B}_{n} \rightarrow \mathcal{M}$ in the sense that

$$
\lim _{n \rightarrow \infty}\left\|x-E_{\mathcal{B}_{n}}(x)\right\|_{2}=0
$$

for all $x \in \mathcal{M}$. Let $\mathcal{R}$ be an intermediate injective von Neumann subalgebra, $\mathcal{A} \subsetneq \mathcal{R} \subset \mathcal{M}$. Take an element $x \in \mathcal{R} \backslash \mathcal{A}$. Then there are numbers $\alpha$ and $\beta$ such that

$$
\|x\|_{2}>\alpha>\beta>\left\|E_{\mathcal{A}}(x)\right\|_{2}
$$

We can find a number $k(>1)$ such that

$$
\left\|E_{\mathcal{B}_{k}}(x)\right\|_{2}>\alpha,\left\|x-E_{\mathcal{B}_{k}}(x)\right\|_{2}<\frac{\alpha-\beta}{2},
$$

since $\lim _{n \rightarrow \infty}\left\|x-E_{\mathcal{B}_{n}}(x)\right\|_{2}=0$, for all $x \in \mathcal{M}$.

Note that

$$
\begin{aligned}
\mathcal{B}_{k}^{\prime} \cap \mathcal{M} & =\left(\left(\mathcal{M}_{1} \otimes \cdots \otimes \mathcal{M}_{k-1}\right) \otimes\left(\otimes_{i \geq k} \mathcal{A}_{i}\right)\right)^{\prime} \cap\left(\left(\otimes_{i<k} \mathcal{M}_{i}\right) \otimes\left(\otimes_{i \geq k} \mathcal{M}_{i}\right)\right) \\
& =\left(\mathcal{M}_{1} \otimes \cdots \otimes \mathcal{M}_{k-1}\right)^{\prime} \cap\left(\mathcal{M}_{1} \otimes \cdots \otimes \mathcal{M}_{k-1}\right) \otimes\left(\otimes_{i \geq k} \mathcal{A}_{i}\right)^{\prime} \cap\left(\otimes_{i \geq k} \mathcal{M}_{i}\right) \\
& =\mathbb{C} I \otimes\left(\otimes_{i \geq k}\left(\mathcal{A}_{i}^{\prime} \cap \mathcal{M}_{i}\right)\right) \\
& \subseteq \mathbb{C} I \otimes\left(\otimes_{i \geq k} \mathcal{A}_{i}\right),
\end{aligned}
$$

where the last equality holds true due to Lemma 2.1. Moreover, by the proof of Lemma 2.4 in CFRW, if $\mathcal{A}$ is a type $I$ von Neumann subalgebra in a finite von Neumann algebra $\mathcal{M}$ and $\mathcal{A}^{\prime} \cap \mathcal{M} \subseteq \mathcal{A}$, then $\left(\mathcal{A}^{\prime} \cap \mathcal{M}\right)^{\prime} \cap \mathcal{M}=\mathcal{M}$.

It implies from Lemma 3.6.5 in [SS] that $E_{\mathcal{B}_{k}}(x)$ is the unique element of minimal $\|\cdot\|_{2}$ in

$$
\begin{aligned}
& \overline{\operatorname{conv}}^{w}\left\{u x u^{*}: u \in \mathcal{U}\left(\mathcal{B}_{k}^{\prime} \cap \mathcal{M}\right)\right\} \\
& \subseteq \overline{\operatorname{conv}}^{w}\left\{u x u^{*}: u \in \mathcal{U}\left(\mathbb{C} I \otimes\left(\otimes_{i \geq k} \mathcal{A}_{i}\right)\right)\right\} \\
&=\overline{\operatorname{conv}}^{w}\left\{u x u^{*}: u \in \mathcal{U}\left(\otimes_{i \geq k} \mathcal{A}_{i}\right)\right\},
\end{aligned}
$$

where $\mathcal{U}(\mathcal{N})$ is the unitary group of von Neumann algebra $\mathcal{N}$. Hence, $E_{\mathcal{B}_{k}}(x) \in$ $\mathcal{R} \cap \mathcal{B}_{k}$. Let us denote $E_{\mathcal{B}_{k}}(x)$ by $y$. Then we have

$\left\|E_{\mathcal{A}}(y)\right\|_{2}=\left\|E_{\mathcal{A}}(y-x)+E_{\mathcal{A}}(x)\right\|_{2} \leq\|y-x\|_{2}+\left\|E_{\mathcal{A}}(x)\right\|_{2}<\frac{\alpha-\beta}{2}+\beta<\alpha<\|y\|_{2}$.

Thus, $y=E_{\mathcal{B}_{k}}(x)$ is not in $\mathcal{A}$. Let $\mathcal{S}$ be the von Neumann subalgebra of $\mathcal{R} \cap \mathcal{B}_{k}$ generated by $y$ and $\mathcal{A}$. Then $\mathcal{S}$ is injective, since $\mathcal{R}$ in injective. Hence, we have $\mathcal{A} \subsetneq \mathcal{S} \subset \mathcal{B}_{k}$. On the other hand, by Lemma 2.5, $\mathcal{A}_{1} \otimes \cdots \otimes \mathcal{A}_{k-1}$ is maximal injective in $\mathcal{M}_{1} \otimes \cdots \otimes \mathcal{M}_{k-1}$. Moreover, $\otimes_{i \geq k} \mathcal{A}_{i}$ is injective. By a theorem in 
[SZ (see also Theorem 2.5 in [Fa]), $\mathcal{A}$ is maximal injective in $\mathcal{B}_{k}$. This leads to a contradiction. Hence, $\mathcal{R}=\mathcal{A}$. The proof is complete.

Popa $[\mathrm{Po}$ showed that the generator masas are asymptotically orthogonal in the free group factor $L_{\mathbb{F}_{n}}$ after tensoring a finite von Neumann algebra (see Section 14.2 in SS ) and an explanation in (CFRW]). Cameron, Fang, Ravichandran, and White CFRW] showed that the radical masa is asymptotically orthogonal in $L_{\mathbb{F}_{n}}$ after tensoring a finite von Neumann algebra (Theorem 6.2 in [CFRW]). Thus, as a consequence of the above theorem, we have the following result, which generalizes the results in $\mathrm{Sh}$ and $\mathrm{CFRW}$.

Corollary 2.7. Let $\mathcal{M}_{i}$ be the group von Neumann algebra of the free group $F_{i}$ generated by $k_{i}(<\infty)$ elements $a_{1, i}, a_{2, i}, \cdots, a_{k_{i}, i}, k_{i} \geq 2$, for $i=1,2, \cdots$. Let $\mathcal{A}_{i}$ be the masa of $\mathcal{M}_{i}$ generated by $a_{1, i}$ or $w_{i}=\sum_{i=1}^{k_{i}}\left(a_{j, i}+a_{j, i}^{-1}\right)$. Then $\mathcal{A}=\otimes_{i=1}^{\infty} \mathcal{A}_{i}$ is a maximal injective subalgebra of von Neumann algebra $\mathcal{M}=\otimes_{i=1}^{\infty} \mathcal{M}_{i}$.

\section{ACKNOWLEDGEMENT}

The author would like to thank Dr. Junsheng Fang for his valuable suggestions.

\section{REFERENCES}

[CFRW] J. Cameron, J. Fang, M. Ravichandran, and S. White. The radical masa in a free group factor is maximal injective. arXiv: 0810.3906v1[math.OA], 21 Oct. 2008.

[Co] A. Connes. Classification of injective factors. Cases $I I_{1}, I I_{\infty}, I I I_{\lambda}, \lambda \neq 1$. Ann. Math. (2), 104(1): 73-115, 1976. MR0454659 (56:12908)

[Fa] J. Fang. On maximal injective subalgebras of tensor products of von Neumann algebras. J. Funct. Anal., 244(1): 277-288, 2007. MR2294484 (2008d:46080)

[Ge] L. Ge. On 'Problems on von Neumann algebras by R. Kadison, 1967'. Acta Math. Sinica, English Series, 19(3): 619-624, 2003. MR2014042 (2005a:46120)

[Ge1] L. Ge. On maximal injective subalgebras of factors. Ad. Math., 118: 34-70, 1996. MR:1375951 (97g:46079)

[GK] L. Ge and R. Kadison. On tensor products for von Neumann algebras. Invent. Math., 123(3): 453-466, 1996. MR.1383957(97c:46074)

[Hou] C. Hou. On maximal injective subalgebras in a $w \Gamma$ factor. Science in China Series A: Mathematics, 51: 2089-2096, 2008. MR2447433(2009i:46114)

[Ka] R. Kadison. Problems on von Neumann algebras. Notes of Baton Rouge Conference, unpublished, 1967.

[KR] R. Kadison and J. Ringrose. Fundamentals of the theory of operator algebras. II. Graduate Studies in Mathematics, 16, Amer. Math. Soc., 1997. MR1468230 (98f:46001b)

[Po] S. Popa. Maximal injective subalgebras in factors associated with free groups. Adv. Math., 50: 27-48, 1983. MR720738 (85h:46084)

[Sh] J. Shen. Maximal injective subalgebras of tensor products of free group factors. J. Funct. Anal., 240(2): 333-348, 2006. MR2261686 (2008g:46109)

[SS] A. Sinclair and R. Smith. Finite von Neumann algebras and masas. London Mathematical Society Lecture Note Series, 351, Cambridge University Press, 2008. MR2433341 (2009g:46116)

[SZ] S. Stratila and L. Zsido. The commutation theorem for tensor products over von Neumann algebras. J. Funct. Anal., 165: 293-346, 1999. MR1698940(2000j:46115)

Department of Mathematics, Louisiana College, Pineville, Louisiana 71359

E-mail address: gao@lacollege.edu 